\title{
Rückblick auf die Anfänge der Schreiberforschung zur Dresdner Instrumentalmusik
}

Der Titel, den mein Vortrag trägt, folgt der mir von der Leitung des Kolloquiums vorgeschlagenen Themenformulierung. Wenn ich selbst versucht wäre, diese Titelfassung um den Zusatz „oder: Jugenderinnerungen eines alten Mannes“ zu erweitern, so drückt sich darin nicht nur ein Kokettieren mit meinem Lebensalter aus - dieser von Wilhelm von Kügelgen entlehnte Titel kennzeichnet auf treffende Weise den Charakter dessen, was ich vortragen möchte. Es geht dabei um einen persönlichen Erlebnisbericht, der weit zurückliegende Arbeitserfahrungen zum Gegenstand hat und der naturgemäß nur solche Dinge berücksichtigen kann, die meine Erinnerung bewahrt hat. Vieles an Details ist mir nicht mehr gegenwärtig, anderes in seinen Fragestellungen fern gerückt. Da viele meiner damaligen Aufzeichnungen (sofern überhaupt noch vorhanden) keine genauen Daten tragen, weiß ich nicht einmal mehr, wann genau und wie oft ich ,vor Ort' in der Landesbibliothek gearbeitet habe; es mögen zwischen Jahresbeginn 1962 und Spätherbst 1964 vier oder fünf Aufenthalte von jeweils ein bis drei Wochen gewesen sein. Was ich noch weiß: Es waren ungemein arbeitsintensive, aber auch sehr schöne und teilweise aufregende Aufenthalte, die ich im Lesesaal in der Marienallee verbracht habe, fürsorglich betreut von den Mitarbeitern der Musikabteilung um Wolfgang Reich (damals noch ganz jung in seinem Amt als Abteilungsleiter) und Lieselotte Willi.

Eine zweite kurze Vorbemerkung sei mir gestattet: Der Charakter des Nachfolgenden als eines persönlichen Erlebnisberichtes bringt es mit sich, dass meine eigenen Arbeiten und damit meine Person über Gebühr in den Vordergrund rücken. Das ist nicht Ausdruck einer unangemessenen Überbewertung der eigenen Leistung! Ich weiß sehr wohl: Wenn meinen damaligen Studien, wie gelegentlich gesagt worden ist, ein gewisser „Pioniercharakter“ zukommt, dann liegt das schlicht daran, dass ich - eher zufällig - derjenige war, von dem Ortrun Landmann eingangs ihrer Studie zu den Dresdner Hofnotisten schreibt:

„Vor mehr als 45 Jahren befaßte sich erstmals ein Autor mit Musikhandschriften der damaligen Sächsischen Landesbibliothek nicht, um die darin enthaltene Musik zu untersuchen, sondern er machte die Überlieferungsgeschichte als solche zum Gegenstand seiner Forschungen."

1 Ortrun Landmann, Über das Musikerbe der Sächsischen Staatskapelle. Drei Studien zur Geschichte der Dresdner Hofkapelle und Hofoper anhand ihrer Quellenüberlieferung in der SLUB Dresden, ${ }^{2} 2010$, S. 122,

https://nbn-resolving.org/urn:nbn:de:bsz:14-qucosa-38515. 
Denn dies ist das aus heutiger Perspektive so verwunderlich Erscheinende: Es gab natürlich Arbeiten, die sich den in der Dresdner Bibliothek überlieferten Werkbeständen einzelner Komponisten widmeten, aber in diesen Untersuchungen spielten Überlieferungs- und Schreiberfragen praktisch keine Rolle. Lediglich Autographe wurden als solche registriert und ansatzweise auch in ihren Merkmalen beschrieben; um die übrigen Schreiber kümmerte man sich nicht, schon gar nicht um die Zuordnung bestimmter Schreiberhände an bestimmte Personen. Als beispielhaft dafür kann Günter Haußwalds Dissertation über Heinichens Instrumentalwerke (mit Werkverzeichnis) von 1937 gelten. Nachdem er die autographe Überlieferung kurz beleuchtet hat, heißt es zu den übrigen Handschriften:

„Bunte, vielgestaltige Schriftformen mit einer verwirrenden Fülle von Einzelzügen und Sonderprägungen weisen die übrigen überlieferten Instrumentalwerke auf. Mitunter jedoch vermutet man den gleichen Schreiber.“2

Durchaus vergleichbar stellt sich die Situation in Hans Rudolf Jungs Pisendel-Arbeit von $1956^{3}$ dar; allerdings erbrachte die nunmehrige Kenntnis der Notenschrift Johann Georg Pisendels die Voraussetzung, um dessen einzigartige Stellung als Schreiber innerhalb des instrumentalen Repertoires insgesamt zu erkennen.

So etwa stellte sich die Situation dar, als ich 1961 mit meinen Arbeiten zur Erforschung der Dresdner Vivaldi-Quellen - zunächst eingegrenzt auf die Handschriften der Konzerte und Sinfonien ${ }^{4}$ begann. (Dass auch die in anderen deutschen Bibliotheken überlieferten Vivaldi-Handschriften Gegenstand der Untersuchungen waren, bleibt hier unberücksichtigt.) Ich war damals im ersten Jahr Assistent bei Rudolf Eller in Rostock, und er war es, der mir dieses Thema für meine Dissertation gestellt hatte; er hatte sich, wie bekannt, in den 1950er Jahren eingehend mit den in Dresden überlieferten Konzerten Vivaldis - aber eben mit deren musikalischen Formungsprinzipien und deren Stilistik - befasst. Natürlich war das, was Rudolf Eller und mir selbst als Ergebnis einer solchen Arbeit vorschwebte, unverkennbar an den Vorbildern orientiert, die die Exponenten der „neuen Bachforschung“, Alfred Dürr und Georg von Dadelsen, mit ihren spektakulären philologischen Studien der späten 1950er Jahre geliefert hatten. Nicht zuletzt gilt dies für das methodische Instrumentarium. All dies bedeutet: Die Zielstellung war eine umfassende Untersuchung der Quellen im Hinblick auf Schreiber, Papiere und archivalische Daten, um so ein Maximum an Informationen über Ursprung, Entstehungszeit und Überlieferungsgeschichte der Handschriften zu gewinnen. (Weitere Aspekte meiner Untersuchungen, so Vergleiche der in Dresden überlieferten Werkfassungen mit denen der konkordanten Überlieferung und speziell die Behandlung

2 Günter Haußwald, Johann David Heinichens Instrumentalwerke, Wolfenbüttel 1937, S. 29 f.

3 Vgl. Hans Rudolf Jung, Johann Georg Pisendel (1687-1755). Leben und Werk, Phil. Diss. Jena 1956 (mschr.).

4 Die Quellen der Sonaten und Trios wurden erst für die Drucklegung der Dissertation in die Untersuchung einbezogen, diejenigen der Vokalwerke konnten überhaupt nur am Rande berücksichtigt werden. Man vergleiche dazu das mit wenigen Bemerkungen zu den Schreibern versehene Verzeichnis der Vokalwerke in der maschinenschriftlichen Fassung der Arbeit (Die deutsche Vivaldi-Überlieferung. Untersuchungen über die in deutschen Bibliotheken handschriftlich überlieferten Konzerte und Sinfonien Antonio Vivaldis, Phil. Diss. Rostock 1965, 2 Bde.), Bd. 1, S. 17 ff. 
der Dresdner Bearbeitungspraxis, bleiben im Kontext der hier zu behandelnden Fragestellungen unberücksichtigt. ${ }^{5}$ )

Bei Lichte besehen stand es um die Voraussetzungen, die ich für eine solche Arbeit mitbrachte, durchaus nicht zum Besten. Nicht nur war ich überhaupt ein ,Anfänger', auf dem Gebiet der historischen Quellenforschung fehlte es mir weitgehend an speziellen Kenntnissen und an jeglicher praktischen Erfahrung.

Meine ersten Arbeitsschritte bestanden darin, die etwa einhundert zur Untersuchung stehenden Notenhandschriften nach signifikanten Quellen- und Überlieferungsmerkmalen zu ordnen. Soweit es die Schreiberhände betraf, konnte ich dies, wenigstens vorläufig, anhand der Fotokopien tun, die für das Rostocker Institut angeschafft wurden, andere Kennzeichen - namentlich solche papierkundlicher Art - waren natürlich nur an den Quellen selbst, das heißt während meiner Besuche in der Bibliothek, auszumachen.

Von den in der Vivaldi-Sammlung auftretenden Schreiberhänden waren nur zwei namentlich identifiziert: diejenige Vivaldis selbst und diejenige Pisendels. In Bezug auf Vivaldi ist dies allerdings dahingehend einzuschränken, dass nur die mit Widmung an Pisendel versehenen Manuskripte als Autographe klassifiziert waren, ${ }^{6}$ andere Handschriften dagegen, in denen ebenfalls Autographe vermutet werden durften, nicht. ${ }^{7}$ In dem Bemühen, über die schriftkundliche Bewertung auch dieser Quellen Klarheit zu gewinnen, habe ich - unter enger Anlehnung an die methodischen Hinweise Georg von Dadelsens ${ }^{8}$ - versucht, die Notenhandschrift Vivaldis in hauptsächlichen signifikanten Merkmalen zu beschreiben. Weiterführende Beobachtungen speziell zur Altersschrift Vivaldis konnte ich später im Nachwort zur Faksimile-Ausgabe der Sammelhandschrift Concerti con molti Istromenti (Mus.2389-O-4) mitteilen. ${ }^{9}$

Wie ich bei der Klassifizierung aller übrigen - das heißt der anonymen - Schreiberhände vorgegangen bin, darf ich hier als bekannt voraussetzen. ${ }^{10}$ Ich habe an diese Schreiber Siglen (Kennbuchstaben) vergeben, die die Häufigkeit ihres Auftretens in der Vivaldi-Sammlung zur Grundlage haben: Mit den Großbuchstaben A bis D wurden diejenigen vier Schreiber versehen, die sich durch die Menge der von ihnen geschriebenen Manuskripte von allen anderen abheben (Schreiber A bis Schreiber D); „neben Pisendel sind es diese vier Schreiber“, so meine in der Dissertation festgehaltene Beobachtung, „die das Bild der Dresdner Vivaldi-Handschriften

5 Das betreffende Kapitel ist nur in der maschinenschriftlichen Fassung der Dissertation (wie Anm. 4) enthalten; vgl. dort Bd. 1, S. 142-232.

6 Vgl. Katalog der Handschriften der Sächsischen Landesbibliothek (vormals Kgl. öff. Bibliothek) zu Dresden, Bd. 4, Leipzig 1923, darin: „Musikalische Originalhandschriften“, bearb. von Arno Reichert, S. 246.

7 Dies betrifft die Handschriften Mus.2389-O-55 (RV 370), ...O-57 (RV 224), ... -77 (RV 585), ...O-101 (RV 260 ) sowie die beiden letzten Werke in der Handschrift Mus.2389-O-4 (RV 540, RV 149).

8 Vgl. Georg von Dadelsen, Beiträge zur Chronologie der Werke Johann Sebastian Bachs, Trossingen 1958, S. 49-68 („Zur Methode“) sowie „Tabellen zur Schrift-Analyse“, nach S. 176 (Tübinger Bach-Studien. 4/5).

9 Antonio Vivaldi, Concerti con molti Istromenti. Faksimiledruck nach der Musikhandschrift Mus.2389-O-4 der Sächsischen Landesbibliothek Dresden, mit einem Nachwort von Karl Heller, Leipzig 1978; vgl. auch die Einleitung zu einer weiteren, 2007 erschienenen Faksimile-Ausgabe dieses Bandes: Antonio Vivaldi, Concerti con molti Istromenti RV 558, 552, 540, 149. Manoscritto Dresden, Sächsische Landesbibliothek - Staats- und Universitätsbibliothek, a cura di Karl Heller, Florenz 2007 (Vivaldiana. Musiche di Antonio Vivaldi in facsimile. 5).

10 Zu den Einzelheiten dessen, was ich im Folgenden referiere, vergleiche man die Darstellung in meiner Rostocker Dissertation von 1965 (wie Anm. 4) bzw. in deren gekürzter Druckfassung (= Heller, Vivaldi 1971). 
wesentlich bestimmen“. Als Schreiber e bis Schreiber m (Kleinbuchstaben) wurden diejenigen Schreiber geführt, deren Notenschrift in mindestens zwei verschiedenen Manuskripten nachgewiesen werden konnte, und dazu trat eine größere Zahl singulärer Schreiber, die als Schreiber x unter Hinzufügung der Signatur-Schlusszahl des betreffenden Manuskripts bezeichnet wurden (der in der Handschrift Mus.2389-O-66 auftretende singuläre Schreiber trägt den Hilfsnamen Schreiber x 66).

Bezugspunkt für die Benennung der Schreiber war also allein ihr Auftreten in der VivaldiSammlung, wie es ja überhaupt zu betonen gilt, dass meine Schreiber-Forschungen allein von dieser einen ,Zelle' (Vivaldi) ausgegangen sind. Anders konnte ich gar nicht vorgehen. Dass ich die Recherchen über die Grenzen der Vivaldi-Sammlung hinaus dann auch auf einen Kreis von Nachbarbeständen, und zwar ausschließlich aus dem instrumentalen Repertoire, ausgedehnt habe, war erst ein zweiter Schritt, um weitere Informationen über die wichtigsten der,Vivaldi-Schreiber zu erhalten. Für die Möglichkeit, eine beträchtliche Zahl von Notenhandschriften aus diesen Beständen - Handschriften mit Werken von Telemann, Fasch, Quantz, Opern-Ouvertüren Händels usw. - einsehen zu können, schulde ich den Mitarbeitern der Musikabteilung besonderen Dank; ihr Verhalten war - was den Zugang zu solchen Bestandskomplexen betrifft - durch außerordentliche Hilfsbereitschaft und Großzügigkeit geprägt.

Auch wenn diese über die Vivaldi-Sammlung hinausgehenden Recherchen in keiner Weise systematisch angelegt sein konnten, vielmehr im Ganzen eher den Charakter von Stichproben hatten, kam ihnen doch naturgemäß eine ganz wesentliche Bedeutung zu im Hinblick auf das, was ich zu den Schreibern ermitteln konnte. In besonderer Weise gilt dies für diejenigen Schreiber, die sich aufgrund der Erscheinungsbilder ihrer Handschriften - „sehr entpersönlichte(r) Schönschriften“, wie Ortrun Landmann sie charakterisiert hat ${ }^{11}$ - sowie der Menge ihrer Schreibarbeiten, größtenteils Stimmen-Abschriften, von vornherein als beruflich arbeitende Notisten einstufen ließen. Es waren dies in erster Linie meine Schreiber A und D (Abb. 1 bzw. 2). ${ }^{12}$

Damit aber tat sich - so schien es - die Chance auf, diese beiden Schreiber namentlich zu identifizieren, denn die Notisten der Hofkapelle waren ja in den erhaltenen Kapellverzeichnissen aufgeführt. Ausgehend von der Prämisse, dass für die Herstellung der betreffenden Notenmanuskripte hauptsächlich das zweite bis vierte Jahrzehnt des 18. Jahrhunderts in Betracht komme, erschien es mir nahezu sicher, dass sich hinter den Schreibern A und D die beiden in den Kapellverzeichnissen aus dem ersten Jahrhundertdrittel geführten Notisten Johann Jacob Lindner und Johann Wolfgang Schmidt verbergen. Vielerlei weitere Beobachtungen und Gedankenoperationen führten mich zu den hypothetischen Zuordnungen Schreiber A = Lindner, Schreiber D = Schmidt. Glücklicherweise bin ich nicht so weit gegangen, in meiner Dissertation die Schreiber-Siglen durch diese Notisten-Namen zu ersetzen, denn wie man weiß, stimmten diese Zuordnungen nicht. Seit Ortrun Landmanns zuerst 1980 bekannt gewordenen Untersuchungsergebnissen ${ }^{13}$ wissen wir,

11 Landmann, Drei Studien 2010 (wie Anm. 1), S. 138.

12 Vgl. zu Schreiber A Notenschrift in A. Vivaldi, Concerto RV 224, Mus.2389-O-57a, http://digital.slub-dresden. de/id311383173. Vgl. zu Schreiber D Notenschrift in A. Vivaldi, Concerto RV 521, Stimmensatz 2, Mus.2389-O54a, http://digital.slub-dresden.de/id311381499.

13 Vgl. Ortrun Landmann, „Dresden, Johann Georg Pisendel und der ,deutsche Geschmack““, in: Die Einflüsse einzelner Interpreten und Komponisten des 18. Jahrhunderts auf das Musikleben ihrer Zeit. Konferenzbericht der 8. Wissenschaftlichen Arbeitstagung Blankenburg/Harz, 27. Juni bis 29. Juni 1980, Blankenburg 1981, S. 20-34, 


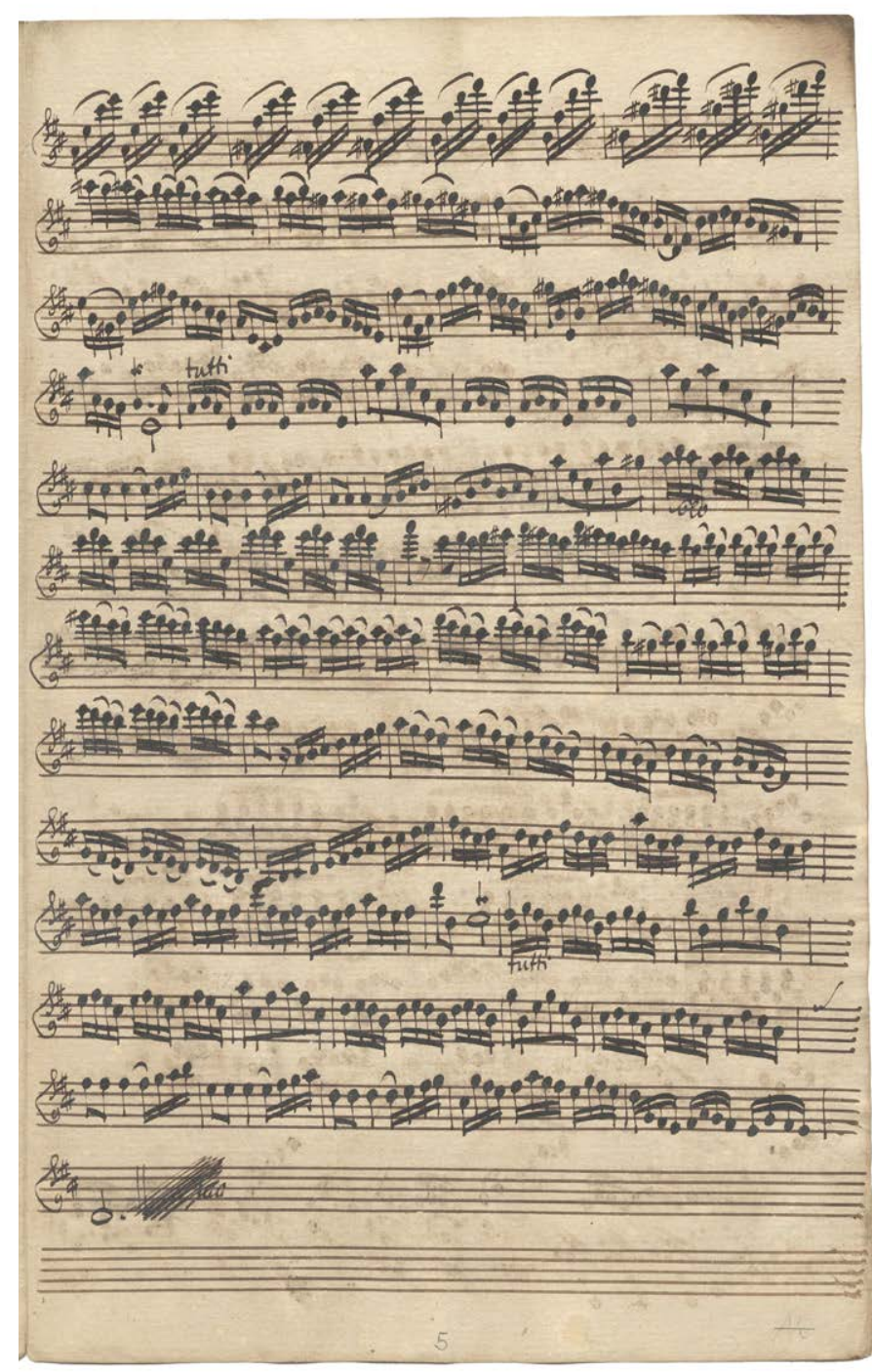

Abb. 1: Notenschrift Schreiber A in A. Vivaldi, Concerto RV 224, letzte Seite der Violino-concertino-Stimme, Mus.2389-O-57a.

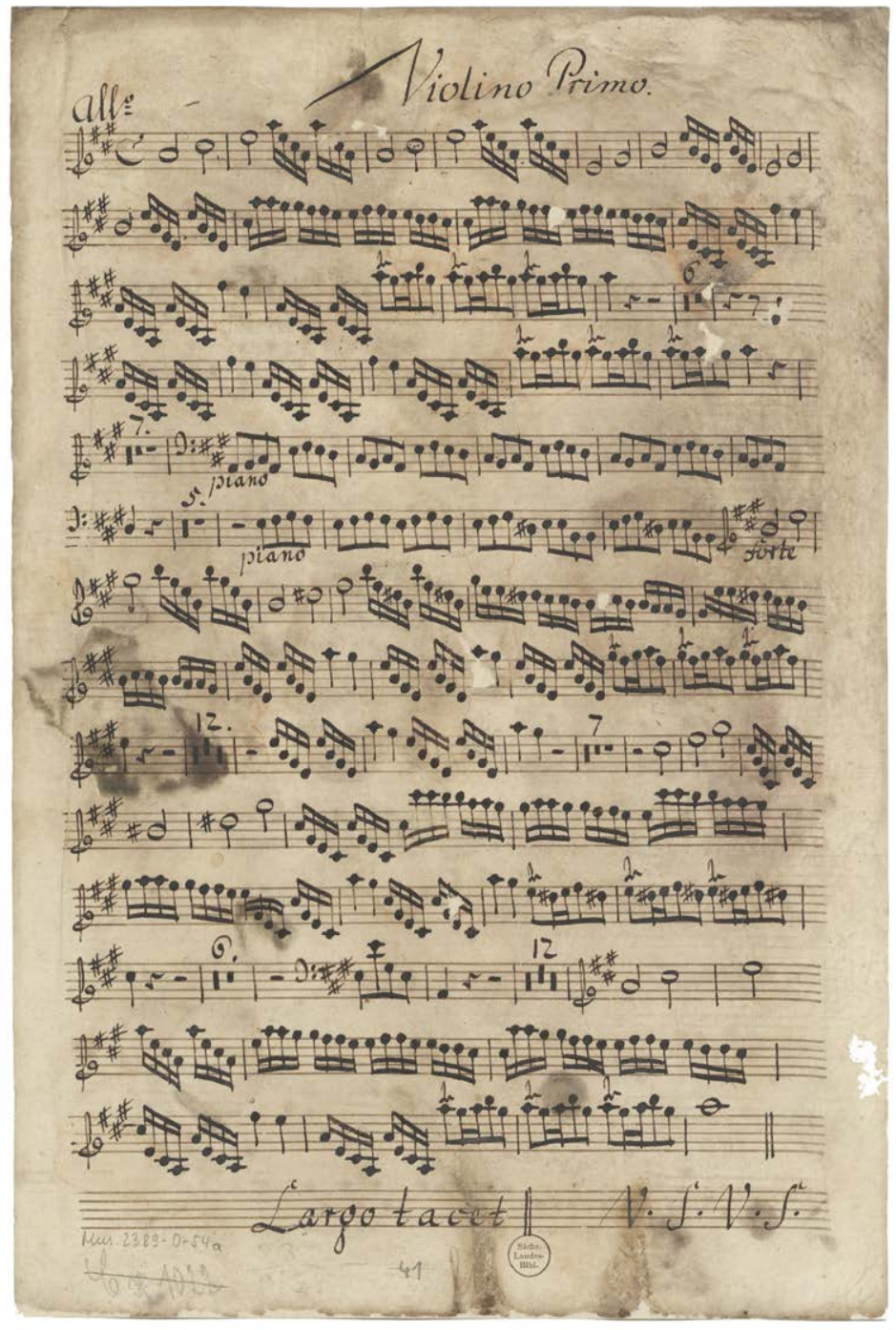

Abb. 2: Notenschrift Schreiber D in A. Vivaldi, Concerto RV 521, Stimmensatz 2, Beginn der zweiten Violino-primo-Stimme, Mus.2389-O-54a.

dass sich hinter meinen Schreibern A und D Vertreter der nachfolgenden Notisten-Generation verbergen. Bis die definitive namentliche Zuordnung gelang - durch wichtige Beobachtungen Manfred Fechners ${ }^{14}$ und durch weitere Forschungen Ortrun Landmanns -, verging nochmals geraume Zeit; dass es sich bei dem als A bezeichneten Schreiber um den Hofnotisten Johann Gottfried Grundig (1706?-1773), bei Schreiber D um den Kapell-Bratschisten und Lohnnotisten Johann Gottlieb Morgenstern (1687-1763) handelt, steht erst seit wenigen Jahren zweifelsfrei fest.

Dass ich mit meinen hypothetischen Zuordnungen einem so gravierenden Irrtum unterlag, hat vielfältige Ursachen. An erster Stelle würde ich aus heutiger Sicht meine fehlenden Einsichten in

bes. S. 27 und S. 32 (Studien zur Aufführungspraxis und Interpretation von Instrumentalmusik des 18. Jahrhunderts, Heft 13).

14 Vgl. zusammenfassend: Manfred Fechner, Studien zur Dresdner Überlieferung von Instrumentalkonzerten deutscher Komponisten des 18. Jahrhunderts, Laaber 1999 (Dresdner Studien zur Musikwissenschaft. 2). Dort sind auch die früheren einschlägigen Arbeiten des Autors dokumentiert. 
die Organisationsstruktur des großen ,Apparates' dieser Hofmusik nennen, speziell in die Praktiken und Regelungen bei der Vergabe und Herstellung von Notenkopien. So wusste ich nichts über das - von Ortrun Landmann jetzt so präzise herausgearbeitete - Nebeneinander von „Hofnotisten“, „Lohnnotisten“, „Notisten im Nebenamt“, Substituten, Lehrlingen usw. Ein entscheidendes Manko war auch die ungenügende Einbeziehung der archivalischen Überlieferung, was in erster Linie der allzu knapp bemessenen Zeit für eigene Archivstudien in Dresden zuzuschreiben war.

Unabhängig davon, dass ich mich bei der personellen Zuordnung der Schreiber A und D auf eine falsche Fährte begab, konnte ich bei der Beschreibung der Notenschriften dieser beiden Notisten ein Moment ins Blickfeld rücken, das sich für die nachfolgenden Forschungen als nicht unerheblich erwies: die Unterscheidung früher und später Schriftformen mit entsprechenden Übergangsmerkmalen und damit das Aufzeigen einer zumindest relativen Chronologie in der individuellen Schriftentwicklung. Die Materialbasis war bei Weitem zu schmal, um alle Stufen und alle Feinheiten zu erfassen, auch ging es natürlich auch hier nicht ohne Irrtümer (zum Beispiel falsche Zuordnungen von Schriftzeugnissen) ab, aber immerhin: Gewisse Grundbeobachtungen konnten formuliert werden, darunter auch solche, die verbindende Merkmale einer von mir damals als „Kopisten-Werkstatt der Dresdner Hofkapelle“ bezeichneten Dresdner ,Schreiber-Schule und damit Momente der Schreiberassimilation betrafen. Manfred Fechner und Ortrun Landmann haben sich später auf sehr viel breiterer Materialbasis der Herausarbeitung der unterschiedlichen Entwicklungsstadien dieser beiden Notisten-Handschriften gewidmet; dass sie dabei teilweise zu divergierenden Ergebnissen gelangten, zeigt die Schwierigkeit solcher Vorhaben, und dies zumal dann, wenn es sich um eng, verwandte', der gleichen Werkstatt angehörende Schreiber handelt.

Mit den von mir als B und C bezeichneten Schreibern (Abb. 3 bzw. 4) wurde eine Kategorie von Notenschreibern ins Blickfeld gerückt, die im Dresdner Quellenbestand an Instrumentalmusik eine nicht geringe Rolle spielt: Schreiber, die das Kopieren von Musikalien nicht als professionell arbeitende Notisten, sondern gleichsam als persönliche Helfer des für das instrumentale Repertoire jahrzehntelang zuständigen Musikers leisteten: Johann Georg Pisendel. ${ }^{15}$ Die Beobachtungen, die ich an den von diesen Schreibern hergestellten Notenkopien Vivaldischer Konzerte machen konnte (bei Schreiber B vierzehn Partituren und drei Stimmensätze, bei Schreiber C zehn Partituren), führten zu der Schlussfolgerung, dass es sich bei ihnen um junge Musiker aus dem Umkreis Pisendels, oder konkreter: um Schüler Pisendels handeln müsse, die unter der Anleitung und Betreuung ihres Mentors als dessen „Privat-Schreiber“"16 arbeiteten. Diese Einstufung der Schreiber B und C ist von der späteren Forschung allgemein akzeptiert worden. Wenn Ortrun Landmann besonders die pädagogische Intention auf Seiten des Lehrers betont hat („das Abschreiben von maßgeblicher neuer Musik“ als Bestandteil der Ausbildung ${ }^{17}$ ), so steht es natürlich außer Frage, dass der Nutzen beidseitig war.

Welche jungen Musiker sich hinter diesen beiden Schreibern verbergen, konnte bislang weder für Schreiber B noch - entgegen einer lange Zeit weitgehend akzeptierten Forschungsmeinung - für

15 Vgl. zu Schreiber B Notenschrift in A. Vivaldi, Concerto RV 384, Mus.2389-O-88,

http://digital.slub-dresden.de/id315431202. Vgl. zu Schreiber C Notenschrift in A. Vivaldi, Concerto RV 199,

Mus.2389-O-78, http://digital.slub-dresden.de/id315370246.

16 Landmann, Drei Studien 2010 (wie Anm. 1), S. 124.

17 Landmann, Dresden, Johann Georg Pisendel und der, deutsche Geschmack'1981 (wie Anm. 13), S. 28. 


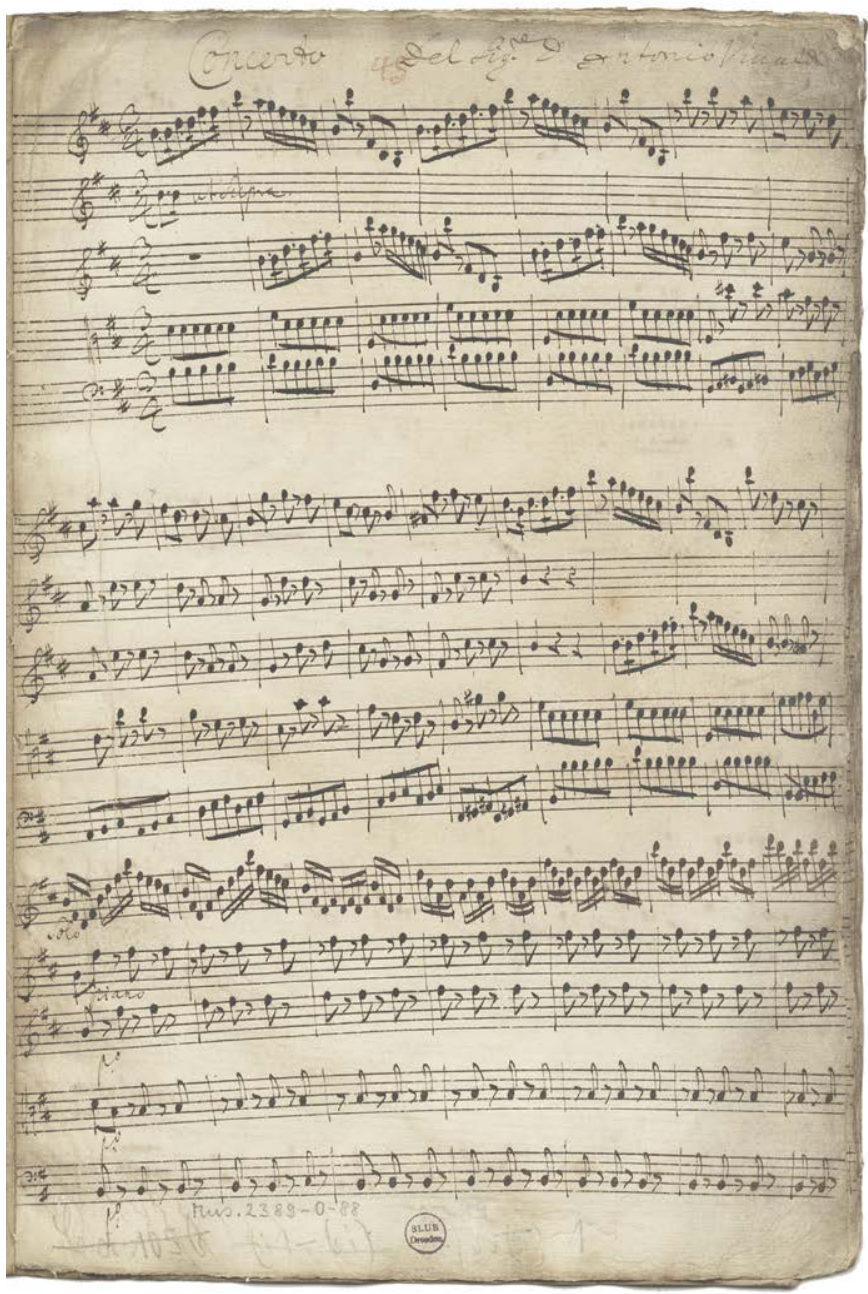

Abb. 3: Notenschrift Schreiber B (mit Eintragungen J. G. Pisendels) in A. Vivaldi, Concerto RV 384, erste Partiturseite, Mus.2389-O-88.

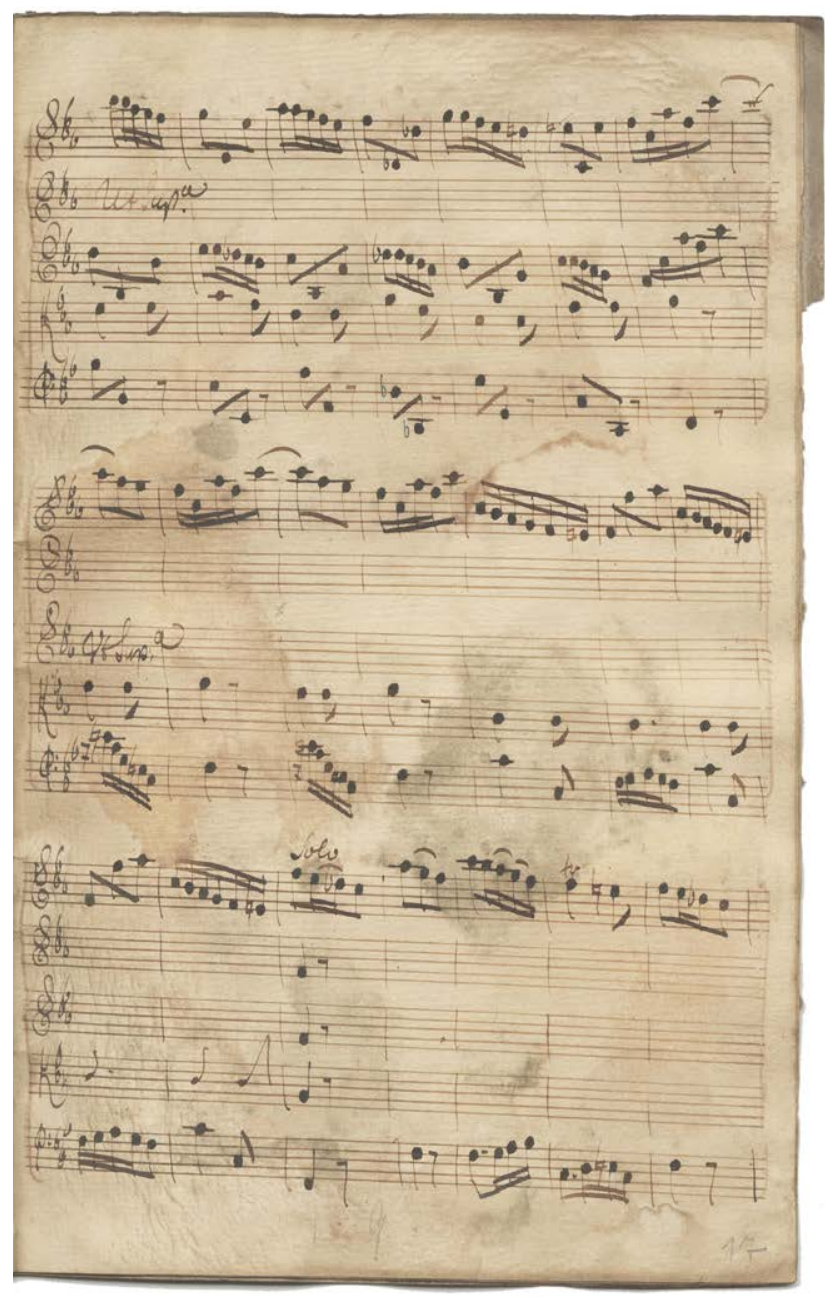

Abb. 4: Notenschrift Schreiber C in A. Vivaldi, Concerto RV 199, Partiturseite von Satz 3, Mus.2389-O-78.

Schreiber C ermittelt werden. Die Notenschrift von Schreiber C, oder genauer: ein Teil der von mir unter Schreiber C gefassten Partitur-Abschriften, wurde durch Horst Augsbach im Zuge seiner Quellenforschungen zu Johann Joachim Quantz (wohl um 1980) dem jungen Quantz zugeordnet, den Augsbach darüber hinaus auch hinter meinem Schreiber f und einigen meiner „singulären Schreiber" zu erkennen glaubte. Während Ortrun Landmann und Manfred Fechner in ihren Publikationen diese Zuschreibungen übernommen haben (erstere mit gewissen Modifikationen ${ }^{18}$ ), konnte ich selbst den Thesen Augsbachs nicht folgen, habe vielmehr noch vor wenigen Jahren die Diskussion zu diesem Fragenkreis als „,noch nicht abgeschlossen "19 ${ }^{19}$ gewertet. Beispielsweise konnte ich nicht nachvollziehen, dass sich, wie Augsbach es sieht, hinter meinem Schreiber C (mindes-

18 Vgl. die Schreiber-Angaben in: Ortrun Landmann: „Katalog der Dresdener Vivaldi-Handschriften und -Frühdrucke“, in: Vivaldi-Studien: Referate des 3. Dresdner Vivaldi-Kolloquiums, Dresden 1981, S. 101-167.

19 Karl Heller, „Pisendels Sammlung Vivaldischer Violinkonzerte“, in: Johann Georg Pisendel - Studien zu Leben und Werk. Bericht über das Internationale Symposium vom 23. bis 25. Mai 2005 in Dresden, hrsg. von Ortrun Landmann und Hans-Günter Ottenberg, Hildesheim u.a. 2010, S. 145-169, hier S. 156 (Dresdner Beiträge zur Musikforschung. 3). 
tens) zwei verschiedene Schreiber verbergen sollen. ${ }^{20}$ Auf die Beweisführung, dass die zehn von mir Schreiber C zugeordneten Vivaldi-Partituren ungeachtet mancher Differenzen im Schriftbild von einem einzigen Schreiber geschrieben sind, war ich damals besonders stolz! So darf ich in den von Mary Oleskiewicz zu diesem Punkt vorgetragenen Ergebnissen eine Bestätigung meiner dazu vertretenen Position erblicken; nach ihren Forschungen handelt es sich weder bei Schreiber C noch bei Schreiber f um Quantz. ${ }^{21}$

Den Schreibern der Kategorie „persönliche Helfer Pisendels“ ist nach jüngeren Erkenntnissen auch der von mir unter x 66 erfasste Schreiber zuzuordnen; bei ihm, der von Ortrun Landmann noch in weiteren Vivaldi-Quellen sowie, unter anderen, in zwei Telemann-Quellen nachgewiesen werden konnte, handelt es sich um den aus Augsburg stammenden Johann Caspar Seyfert, der von 1720 bis 1723 Pisendels Schüler in Dresden war. ${ }^{22}$

Auf die neun von mir mit den Kleinbuchstaben e bis $\mathrm{m}$ versehenen Schreiber will ich nicht weiter eingehen; nur zwei knappe Informationen seien hier eingefügt. Schreiber e (Abb. 5), nach meiner damaligen Beobachtung eindeutig ein Schreiber italienischer Herkunft und, wie sich aus autographen Eintragungen in seinen Kopien schließen ließ, ein Kopist aus dem unmittelbaren Umfeld Vivaldis, wurde vor zwei Jahrzehnten durch Paul Everett hypothetisch als Vivaldis Vater Giovanni Battista Vivaldi identifiziert. ${ }^{23}$ Schreiber 1 ist, wie Ortrun Landmann nachweisen konnte, der Notist, den ich hinter Schreiber A vermutet hatte: Johann Jacob Lindner, „Notist bey der Capelle" schon ab circa 1680. ${ }^{24}$

Eine wesentliche Zielstellung meiner Studien bestand - gerade auch nach den Vorstellungen Rudolf Ellers - darin, nähere Aufschlüsse über die Entstehungszeiten der Dresdner Vivaldi-Quellen zu gewinnen, und im Blick darauf erschien es unabdingbar, das diesbezügliche Informationspotential der papierkundlichen Daten zu nutzen. Nun war aber auf diesem Gebiet die Ausgangslage in keiner Weise besser als auf dem der Schreiberforschung: Es gab keine Erhebungen zu Wasserzeichen in den Notenpapieren dieses Dresdner Repertoires. So habe ich gleich bei meinen ersten Arbeitsaufenthalten in Dresden (und ebenso auch in Schwerin und Rostock) von den am häufigsten vorkommenden Papieren Wasserzeichen-Pausen gezeichnet und diese an den Papierforscher Wisso Weiß, damals in Greiz als Leiter des dortigen Deutschen Papiermuseums ansässig, mit der Bitte um Beschreibung und Bestimmung geschickt; auch zu einem persönlichen Gespräch war ich 1963 in Greiz. Wisso Weiß war in den späten 1950er Jahren in unserem Fach bekannt geworden als Autor des Katalogs Papier und Wasserzeichen der Notenhandschriften von Johann Sebastian Bach, der damals nur erst in maschinenschriftlicher Form vorlag. Die Angaben, die

20 Vgl. Horst Augsbach, Thematisch-systematisches Werkverzeichnis Johann Joachim Quantz (QV), Stuttgart 1997, S. XVIII.

21 Der betreffende Tagungsbeitrag „Quantz als Kopist, Komponist und Vermittler von Dresdener Repertoire: Rheinsberg, Bayreuth, Berlin und seine europäische Reise“ ist nicht im vorliegenden Band enthalten.

22 Angaben nach Landmann, Drei Studien 2010 (wie Anm. 1), S. 181; zu Seyfert vgl. auch Kai Köpp: Johann Georg Pisendel (1687-1755) und die Anfänge der neuzeitlichen Orchesterleitung, Tutzing 2005, S. 416.

23 Vgl. Paul Everett, „Vivaldi’s Italian Copyists“, in: Informazioni e studi Vivaldiani. Bollettino annuale dell' Istituto Italiano Antonio Vivaldi, 11 (1990), S. 27-88, bes. S. 34 ff. Bei Everett trägt Schreiber e die Bezeichnung „scribe 4“. Vgl. zu Schreiber e Notenschrift in A. Vivaldi, Concerto RV 189, erste Seite der Violino Principale, Mus.2389-O66a, http://digital.slub-dresden.de/id311387365.

24 Landmann, Drei Studien 2010 (wie Anm. 1), S. 163. 


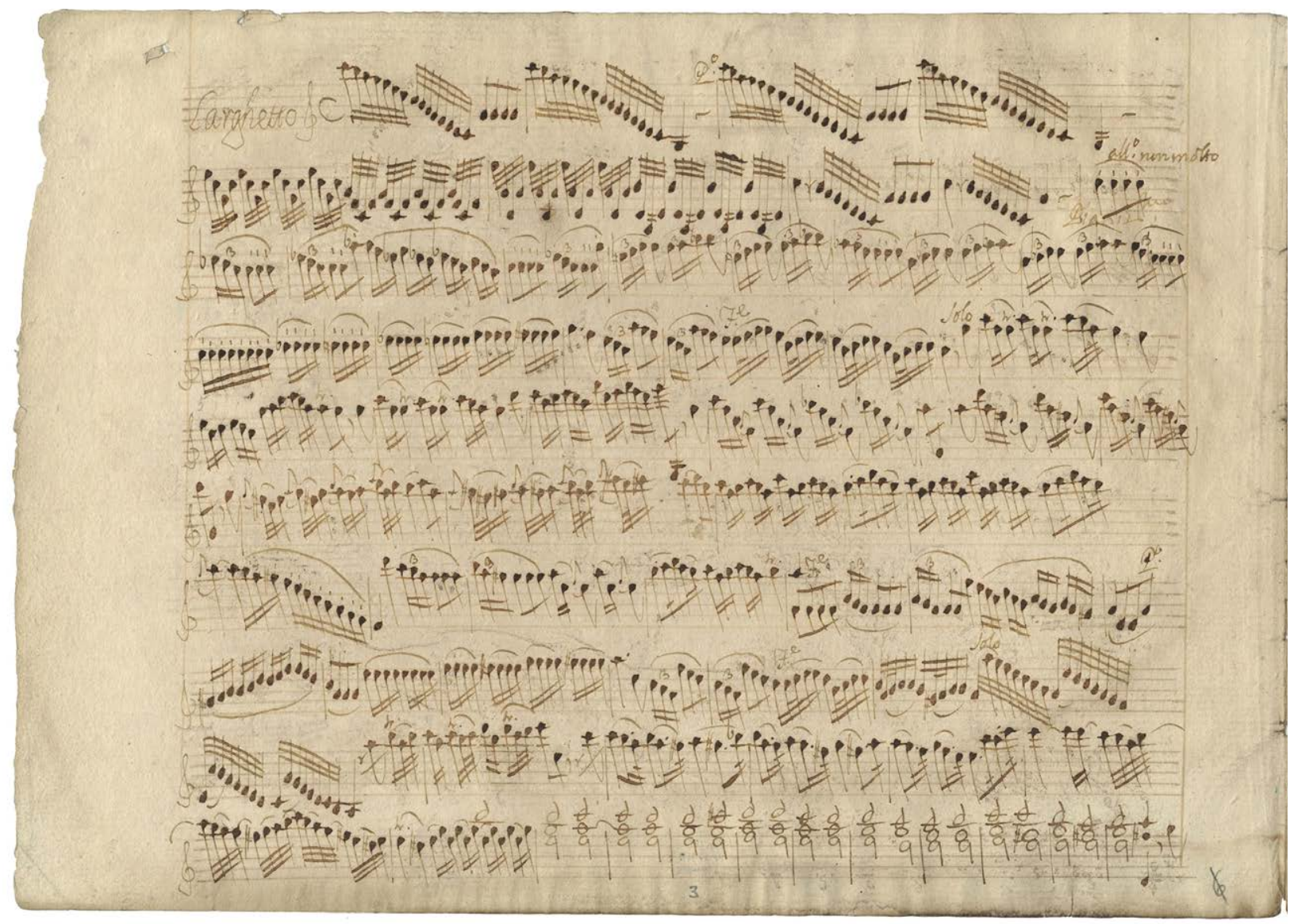

Abb. 5: Notenschrift Schreiber e in A. Vivaldi, Concerto RV 189, erste Seite der Violino-principale-Stimme, Mus.2389-O-66a.

Weiß zu Herkunft und Entstehungszeitraum der von mir eingesandten Zeichen machen konnte, waren weniger ergiebig im Hinblick auf ,punktgenaue ' Datierungen der betreffenden Handschriften als erhofft; die aus den Papierbefunden sowie aus der Kombination von Papier- und Schreiberbefunden erwachsenen Informationen erbrachten aber mittelbar wertvolle Hinweise zur Verortung mancher Handschriften oder Handschriftengruppen. Ich habe die diesbezüglichen Befunde in einem Exkurs „Die Haupt-Wasserzeichen in den Dresdner Vivaldi-Handschriften“ zusammengefasst und im thematischen Quellenkatalog zu jeder Handschrift auch das bzw. die Wasserzeichen angegeben. Auf diese Weise wurden wichtige Wasserzeichen der Sammlung - seien es die „tre lune“ der venezianischen Papiere, die Zeichen der Schuchart-Papiere aus der Papiermühle an der Weißeritz oder der "gekrönte Doppeladler mit Herzschild“ - erstmals als ,Erkennungsmarken benannt und beschrieben (leider ohne Beigabe von Abbildungen) und mit ihren Konnotationen ins Bewusstsein gerückt. Man darf gespannt darauf sein, welchen Wissenszuwachs der Gesamtkatalog der Wasserzeichen des Schrank-II-Bestandes erbringen wird.

Als ein letzter Aspekt sei der Problemkreis ,früheste Überlieferung des Instrumentalmusikrepertoires' kurz angesprochen. Die in Julius Rühlmanns Studie Antonio Vivaldi und sein Einfluß auf Joh. Seb. Bach von 1867 enthaltene Schilderung über „die Auffindung“ eines reichen Bestandes an Instrumentalkompositionen aus dem 18. Jahrhundert „in einem Notenschranke der katho- 
lischen Hofkirche ${ }^{\text {“25 }}$ hatte mich spontan an die auf den Musikalien-Umschlägen angebrachten Titel-Etikette mit der Aufschrift „Schranck No: II“ denken lassen, und so richtete sich mein Interesse darauf, möglichst viel über diesen Schrank in Erfahrung zu bringen. Die Ergebnisse meiner Beobachtungen - am ausführlichsten niedergelegt in der maschinenschriftlichen Fassung der Dissertation ${ }^{26}$ - müssen hier nicht referiert werden; sie betrafen ebenso die Anordnung der Notenbestände in diesem Schrank (mit nicht weniger als 38 für mich nachweisbaren Fächern) wie den Zusammenhang zwischen der Schrank-Nummerierung und dem Signaturen-System der Königlichen Privatmusikaliensammlung, und sie betrafen nicht zuletzt die Frage des Zeitpunktes, zu dem die Archivierung dieses Repertoires erfolgte. Hier geht es mir nur um wenige Bemerkungen zu dem Fragenkreis, der die ursprünglichen Besitzverhältnisse an den um 1765 in besagtem Schrank archivierten Notenbeständen betrifft.

Eine Frage, die sich mir angesichts der im Schrank II gelagerten Vivaldiana verständlicherweise stellte, lautete: Waren diese Handschriften bereits vorher an einer Stelle vereinigt, oder wurden sie erst im Zuge dieser Archivierung aus verschiedenen Teilbeständen zusammengeführt? Ausgehend von dem höchst unterschiedlichen Charakter der Vivaldi-Quellen war ich - zugegebenermaßen von einer vergleichsweise ,naiven' Herangehensweise her - bezüglich der Vivaldi-Bestände zu der Auffassung gelangt, dass, die Besitzverhältnisse betreffend, wohl ein Unterschied bestanden haben müsse zwischen den von bezahlten Notisten gefertigten Aufführungsmaterialien einerseits und solchen Manuskripten, die unstreitig persönlicher Besitz Pisendels gewesen sein mussten, andererseits. Letzteres sah ich als gegeben an zum Beispiel bei den autographen Partituren mit persönlicher Widmung des Komponisten an Pisendel, aber auch bei den zahlreichen von diesem selbst - zu einem großen Teil während seines Venedig-Aufenthalts 1716/17 - gefertigten Partitur-Abschriften Vivaldi'scher Konzerte, Sinfonien und Sonaten. Diese (und nur diese!) Teile der Vivaldi-Sammlung mussten - so meine dazu formulierte Position - mit dem durch Maria Josepha veranlassten Ankauf der „musikalischen Hinterlassenschaft“ Pisendels in den Besitz des Hofes gelangt und bei der Schrank-Archivierung mit den Vivaldiana der kapelleigenen Musikalien vereinigt worden sein.

Der Grund, weshalb ich auf meine damaligen Überlegungen zurückkomme, ist der, dass diese Sichtweise einen erheblichen Einfluss auf nachfolgende Darstellungen zur Überlieferungsgeschichte erlangt hat. So hat namentlich Manfred Fechner meine These aufgegriffen und im Blick auf das gesamte Schrank-Repertoire gleichsam verabsolutiert. ${ }^{27}$ Ich bin also, wenn man so will, ,verantwortlich` für diese über geraume Zeit tradierte Lesart der frühesten Überlieferungsgeschichte des Instrumentalrepertoires - eine Lesart, die, wie Kai Köpp einsichtig gemacht hat, den tatsächlichen historischen Gegebenheiten indes nicht gerecht wird. Vielmehr ist davon auszugehen, dass es eine „Trennung der erhaltenen Musikalien aus ,Schranck No: II.' in Kapellrepertoire einerseits und Pisendels ,Privatsammlung`andererseits“ nicht gegeben hat. Köpp konstatiert:

„Sowohl die Abschriften der besoldeten Notisten als auch diejenigen Musikalien, die Pisen-

27 Vgl. Fechner, Studien 1999 (wie Anm. 14), S. 10-24. 
del selbst geschrieben hat, waren nämlich in einer einzigen, umfangreichen Repertoirebibliothek zusammengefasst, die als Teil der Erbmasse Pisendels angesehen wurde. ${ }^{\text {“28 }}$

Ungeachtet dieser veränderten Sichtweise behält indes, wie ich denke, eine von mir im Blick auf die Vivaldiana getroffene Feststellung ihre Gültigkeit: Bei Weitem nicht alle in Schrank II überlieferten Konzerte und Sinfonien des Venezianers sind in Dresden tatsächlich aufgeführt worden und damit dem Kapell-Repertoire im buchstäblichen Sinne zuzurechnen. Dies erhellt daraus, dass ein überaus hoher Prozentsatz der Werke - auf die Konzerte und Sinfonien bezogen etwa die Hälfte - nur in Partituren vorliegt. Auch wenn in dem einen oder anderen Falle mit dem Verlust des Stimmenmaterials gerechnet werden muss - an diesem so auffälligen Gesamtbild würde sich durch etwaige kleinere Korrekturen kaum etwas ändern. Wie sich in dieser Hinsicht die Situation bei anderen Bestandskomplexen darstellt, werden die Untersuchungen des Schrank-II-Projektes im Detail erweisen.

Meine Schreiber-Untersuchungen an den Vivaldi-Handschriften konnten nur ein erster Anfang zur Erforschung der Dresdner Schreiber sein, ein erstes Herantasten an ein Forschungsfeld, zu dessen umfassender und effektiver Bearbeitung es ganz anderer Voraussetzungen bedurfte und bedarf. Dass meine Arbeit eine Reihe von Grundbeobachtungen zur Schreiber- und Überlieferungssituation insgesamt bereitstellen und eine gewisse Initialwirkung für weitere Forschungen in dieser Richtung erlangen konnte, war schon mehr, als man von einer dem Gegenstand nach so eingegrenzten Studie unbedingt erwarten konnte. Wie es von den 1970er Jahren an weiterging mit der Dresdner Schreiberforschung, ist nicht mehr Gegenstand dieses Vortrages. Alle wichtigen Arbeiten und viele Einzelergebnisse dazu sind jetzt dokumentiert in Ortrun Landmanns wiederholt genannten Drei Studien, einer eindrucksvollen Zusammenschau eigener und fremder Forschungsergebnisse aus mehr als vier Jahrzehnten, vermehrt um vielerlei neue „Erkenntnisse und Erkenntnishypothesen ${ }^{\text {“29 }}$ der Autorin und - besonders wichtig - ergänzt durch einen Abbildungsteil mit mehr als 200 Dokumenten und Kopialien der Dresdner Hofnotisten. Der Wert dieser Studien als Arbeitsinstrument und Diskussionsgrundlage für künftige Forschungen zur Dresdner Musikaliensammlung kann kaum hoch genug veranschlagt werden.

28 Köpp, Pisendel 2005 (wie Anm. 22), S. 404.

29 Landmann, Drei Studien 2010 (wie Anm. 1), S. 124. 